\title{
Collagen I and III and metalloproteinase gene and protein expression in prostate cancer in relation to Gleason score
}

Antonio H. Duarte, Sicilia Colli, Jorge L. Alves-Pereira, Max P. Martins, Francisco J. B. Sampaio, Cristiane F. Ramos

Urogenital Research Unit, State University of Rio de Janeiro, Rio de Janeiro, RJ, Brazil

ABSTRACT

Purpose: To evaluate if the expression of metalloproteinase, collagen I and III are related to Gleason score, preoperative PSA and pathological stage in prostate cancer.

Materials and Methods: Our study group included radical prostatectomy specimens of 33 patients with prostatic adenocarcinoma who underwent surgery from 2001 to 2009. Patients were divided into 3 groups: Gleason score $=6$ (13 patients), Gleason score $=7$ (10 patients), Gleason score $\geq 8$ (10 patients). The control group included prostates of patients submitted to cystoprostatectomy and benign prostatic tissues adjacent to the cancer area. Specific areas of tissues were selected under microscope and further processed for collagen I and III analysis by real time PCR. In addition, 10 deparaffined sections of each group were used to evaluate collagen I, III and metalloproteinase immune expression. The results were correlated with Gleason score, preoperative PSA and pathological stage.

Results: We found significant difference in both collagen I and III gene expression between benign and tumoral areas in the prostate samples from Gleason score $=6$ (collagen $\mathrm{I}=0.4 \pm 0.2$ vs $5 \pm 2.4, \mathrm{p}<0.05$; collagen $\mathrm{III}=0.2 \pm 0.06$ vs $0.7 \pm 0.1$, $\mathrm{p}<0.05$ ) and Gleason score $\geq 8$ (collagen $\mathrm{I}=8 \pm 3.4$ vs $1.4 \pm 0.8, \mathrm{p}<0.07$; collagen $\mathrm{III}=1.8 \pm 0.5$ vs $0.6 \pm 0.1, \mathrm{p}<0.05$ ). There was no correlation of collagen expression with Gleason score, preoperative PSA or pathological stage. There was a positive correlation between metalloproteinase expression and Gleason score $\left(\mathrm{r}^{2}=0.47\right)$. Conclusions: The positive correlation between metalloproteinase expression and Gleason score suggests that metalloproteinase could be a promising factor to improve Gleason score evaluation. Its expression and regulation do not seem to be related with collagen degradation.

\section{ARTICLE INFO}

\section{Key words:}

Prostate cancer;

Prostatic Neoplasms; Collagen;

metalloproteinase;

Gleason score

Int Braz J Urol. 2012; 38: 341-55

Submitted for publication:

July 29, 2011

Accepted after revision:

November 29, 2011

\section{INTRODUCTION}

Prostate cancer is by far the most frequently diagnosed cancer in American males and the second leading cause of cancer deaths in that population. In Brazil, prostate cancer is also the second cause of death in males, being the most frequent cancer after skin tumors. Thus, the progression of prostate cancer from histologic cancer to clinically detectable and metastasizing cancer is of utmost importance. 
Histologically, the prostatic acinus is lined with secretory (typically cuboidal to columnar) luminal epithelial cells and a discontinuous layer of basal cells and is embedded in a fibromuscular stroma (1).

Studies of human breast, colon, and prostatic cancer specimens have identified activated stromal cell phenotypes, modified extracellular matrix (ECM) composition, and increased microvessel density, exhibiting biological markers consistent with what happens in stroma at wound repair site (2-4). Reactive stroma in cancer is composed of fibroblasts, myofibroblasts, endothelial cells, and immune cells. It seems that stromal components play important role in the tumor progression by stimulating the angiogenesis and by promoting cancer cell survival, proliferation, and invasion (5).

All cells included in tumor stroma would potentially affect tumor genesis, however, myofibroblasts are of particular interest. They are activated stromal cells typically found at site of pathologic tissue remodeling $(4,6,7)$. Myofibroblasts in reactive stroma synthesize ECM components such as collagen I, collagen III, fibronectin, tenascin, and versican (8). In addition, myofibroblasts express proteases, fibroblast activation protein (FAP), and matrix metalloproteinases (MMPs). These elements cause remodeling of ECM and basement membrane and can stimulate cancer cell growth and migration (9). Indeed, some MMPs such as type 1, 2 and 9 have been related to prostate cancer $(10,11)$.

The concept that the microenvironment is crucial for the maintenance of cellular functions and tissue integrity suggests that a cancer-induced change in the stroma may contribute to cancer invasion (12). Based on this fact the aim of this study was to evaluate if the expression of metalloproteinase, collagen I and III could be related with Gleason score, PSA and pathological stage.

\section{MATERIAL AND METHODS}

Our study group included radical prostatectomy specimens of 33 patients with prostatic adenocarcinoma who underwent radical surgery from 2001 to 2009. Such patients were divided into 3 groups according to the Gleason score: Gleason score $=6$ (13 patients), Gleason score $=7$ (10 pa- tients), Gleason score $\geq 8$ (10 patients). Since the Gleason score is a combination of primary and secondary grades (or patterns) in biopsy or surgical specimen both grades areas were used in all analysis. The prostate from patients with bladder cancer submitted to cystoprostatectomy were used as a control group. Benign prostatic tissues adjacent to the cancer area in the different Gleason grades were also used as a second control group. The Gleason score classification was performed by a same group of pathologists with large experience in prostate cancer. The study was approved by the internal review board of the institution and all patients agreed to sign the informed consent.

The criterion of tumor recurrence was defined as PSA $\geq 0.2 \mathrm{ng} / \mathrm{mL}$ according to the AUA recommendation. The postoperative follow up period varied from 1 to 62 months. The mean follow up was 20.5 months and the median was 18.0 months. The age of patients with prostate cancer ranged from 45 to 75 years (median age 63.3), and the age of patients submitted to cystoprostatectomy ranged from between 58 to 75 years (median 67.7 years).

The paraffin-embedded blocks of the different groups were used to determine the expression of collagen I, III and metalloproteinase by immunohistochemistry. The adenocarcinoma and benign areas were selected from tissues under microscope analysis and further used to determine gene expression of collagen I and III by real time PCR.

RNA was extracted using the recoverall total nucleic acid isolation kit (Applied biosystems, Carlsbad, CA, USA) according to the manufacturer's protocol. All samples were treated with DNAase Free reagent (Invitrogen, CA, USA) following the manufacturer's protocol for elimination of residual DNA. Then, a hundred nanograms of RNA were used to evaluate the gene expression of collagen I and III by real time PCR using the Express one-step SYBR GreenER kit (Invitrogen, CA, USA). The reactions were performed in triplicate and normalized by $\beta$-actin gene expression. The primer sequences used are described below: Collagen I: sense: gtgctaaaggtgccaatggt; antisense: accaggttcaccgctgttac. Collagen III sense: ctggacctcagggacc; antisense: gttcccaggttttccat. $\beta$-actin: Sense: ccagctcaccatggatga; antisense: acgatggaggggaagac. 
For immunohistochemistry analysis, 10 deparaffined sections of each group were hydrated, treated with buffer TRIS-EDTA (pH 9.0) overnight at $60^{\circ} \mathrm{C}$ (collagen I and III) or citrate buffer, pH6.0, for $20 \mathrm{~min}$ at $600 \mathrm{C}$ (metalloproteinase 13) for antigen retrieval, and then treated with 3\% hydrogen peroxide solution in methanol for 10 min to block endogenous peroxidase activity. These steps were followed by washing the sections in PBS and subsequently incubated $10 \mathrm{~min}$ at room temperature with $10 \%$ goat serum to block unspecific binding. The sections were then incubated for $2 \mathrm{~h}$ at room temperature with Collagen I and III primary antibodies (Abcam, Cambridge, MA, USA) or overnight at $4^{\circ} \mathrm{C}$ with metalloproteinase 13 antibody (Millipore, Billerica, MA, USA) diluted in PBS with 1\% BSA. Sections were then washed in PBS and incubated at room temperature for 20 min with biotinylated secondary antibody followed by incubation at room temperature for $10 \mathrm{~min}$ with streptavidin-peroxidase conjugate (Histostain-Plus Kit, Invitrogen, CA, USA). Sections were washed in PBS, then revealed by treating with liquid diaminobenzidine (Histostain-Plus Kit, Invitrogen, CA, USA), and then counterstained with hematoxylin. The negative controls were processed by replacing the primary antibody with PBS and no indication of staining was observed.

Images were digitized using an Olympus DP70 (12.5 megapixels, Tokyo, Japan) video camera coupled to a BX51 Olympus light microscope (Tokyo, Japan), which transferred all images captured to a microcomputer. The quantitative analysis was performed at a final magnification of 200x using a software Image-Pro Plus (4.5.0.29, Media Cybernetics, Inc, Bethesda, MD, USA) and a technique based on color segmentation (13).

\section{Statistical analysis}

The tumor free survival time was determinate by Kaplan-Meier analysis. The odds ratio and its confidence interval of 95\% were analyzed for each parameter studied (stage, PSA, Gleason). Statistical significance of gene expression and immunohistochemistry observations were determined by Student's-t-test. Statistical significance among cystoprostatectomy and benign areas from different Gleason scores was determined by ANOVA followed by Newman-Keuls test. All results were presented as mean \pm SEM. The level of significance was set at $\mathrm{p}<0.05$.

\section{RESULTS}

Figure- 1 shows the results of PSA free percent survival in relation to pathological stage, preoperative PSA and Gleason score for 5 years.

Figure 1 - Kaplan-Meier disease-free survival curves. Pathological stage (A), PSA (B), Gleason scores (C).

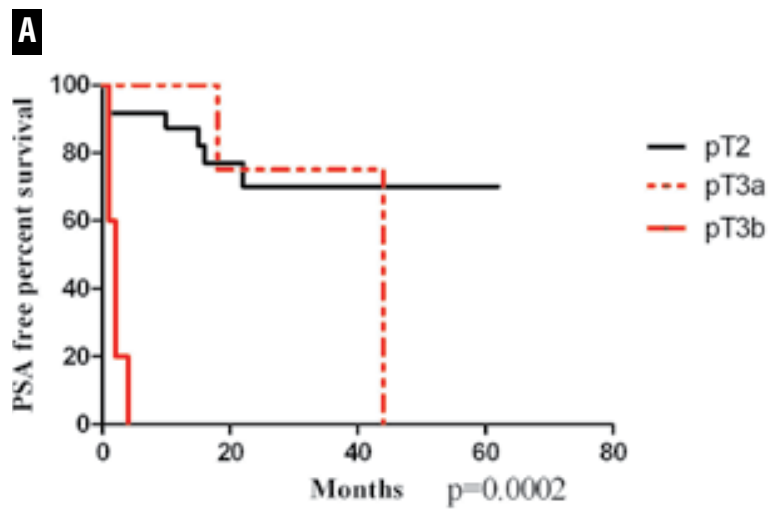

B

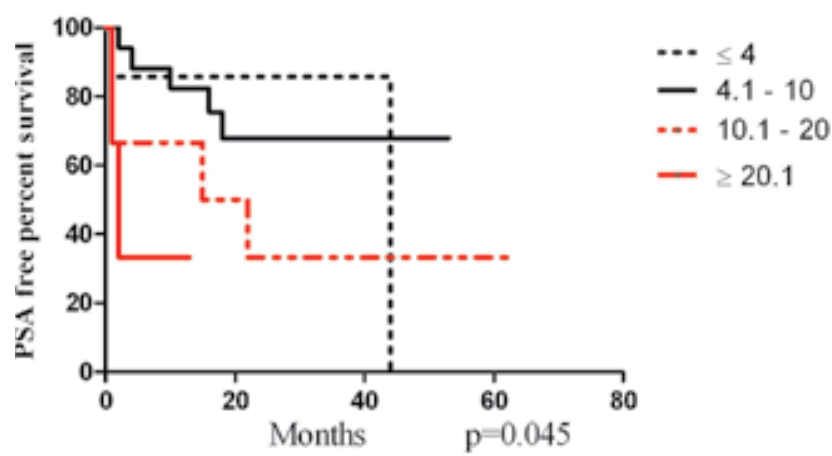

C

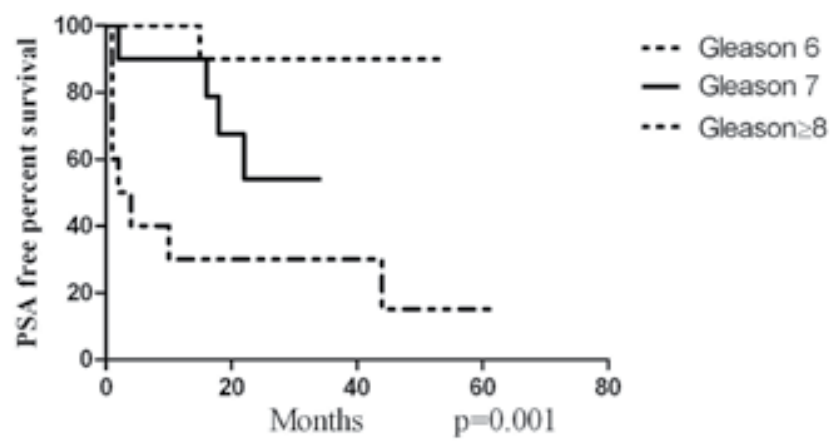


The real time PCR analysis of both collagen I and III gene expression showed that there is a significant difference $(p<0.05)$ between tumor and benign areas in the prostate samples from Gleason 6 and $\geq 8$. The samples of Gleason 7 showed no statistical difference between the tumor and benign areas (Figure-2). We found no correlation between collagen gene expression and Gleason score in tumor areas (Figure-3).

The immunohistochemistry analysis by color segmentation technique showed that collagen I and III expression was significantly reduced in prostate

Figure 2 - Gene expression of collagen I and III in the benign (white bar) and tumor (black bar) areas of prostate of Gleason score $=6(A, B)$, Gleason score $=7(C, D)$ and Gleason score $\geq 8(E, F) . \beta$ actin was used as an internal control. Data are represented as means \pm SEM. Sample numbers were 13 for Gleason score $=6$; 10 for Gleason score $=7$ and 10 for Gleason score $\geq 8$. Different letters mean statistical significance evaluated by Student's-t-test.
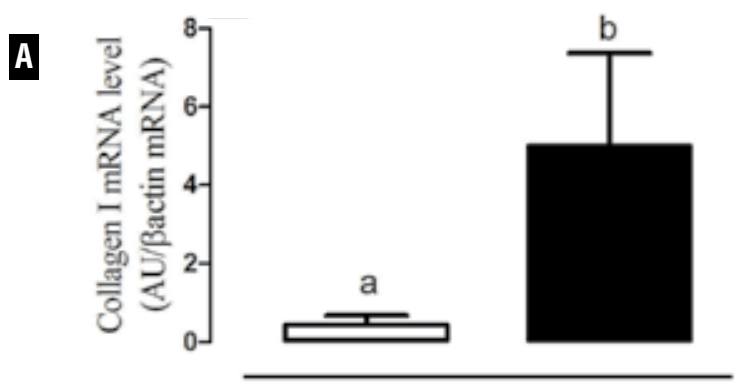

Gleason score $=6$
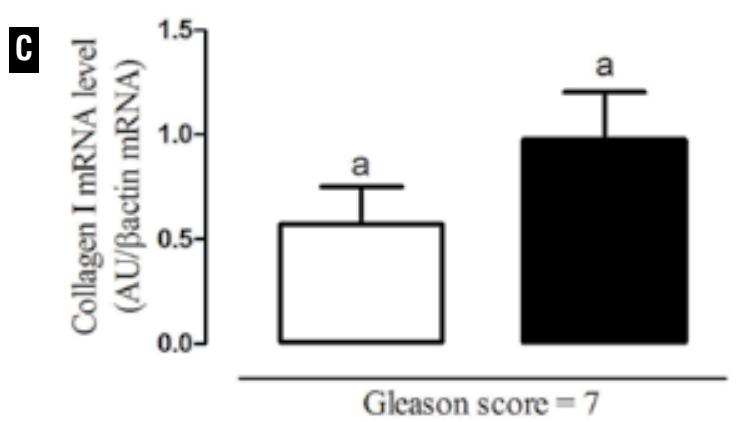

日

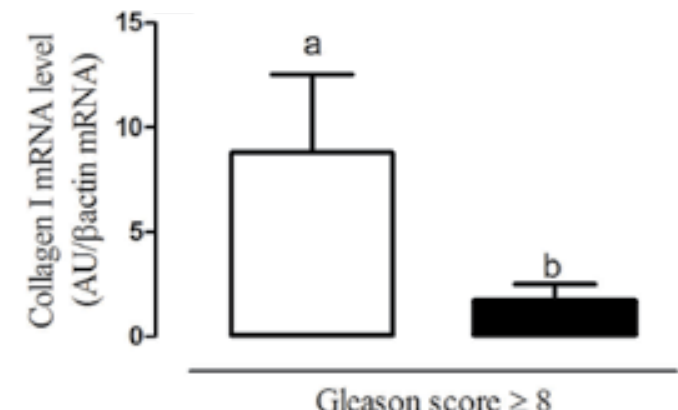

B
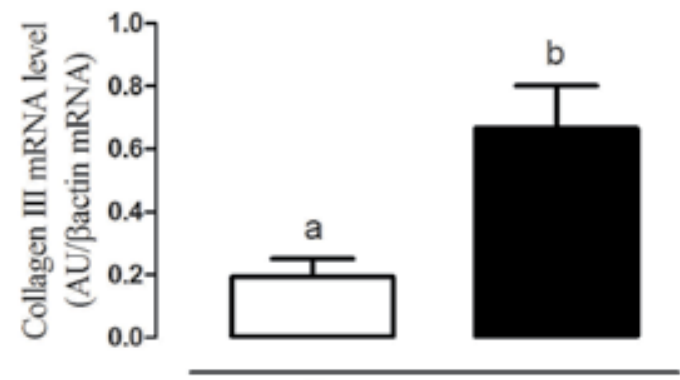

Gleason score $=6$

D
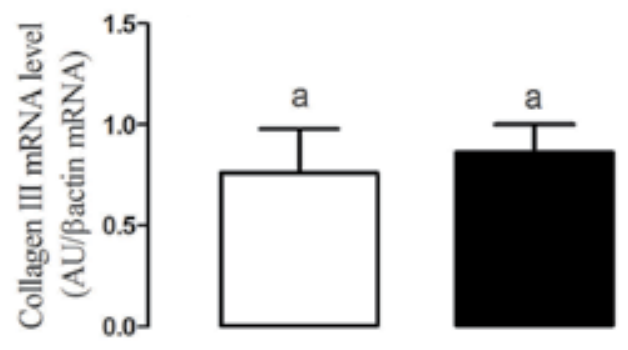

Gleason score $=7$

F
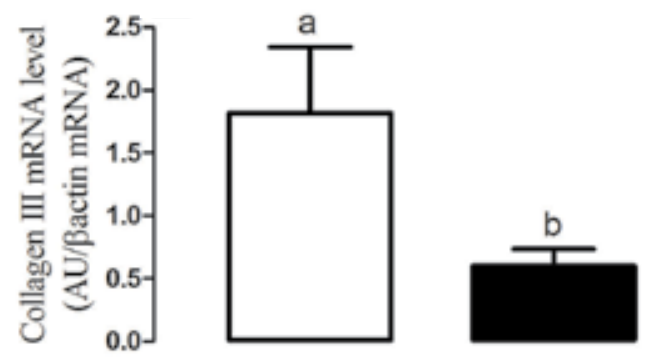

Gleason score $\geq 8$ 
Figure 3 - Linear regression analysis between collagen expression and Gleason score. Collagen I (A) Collagen III (B).
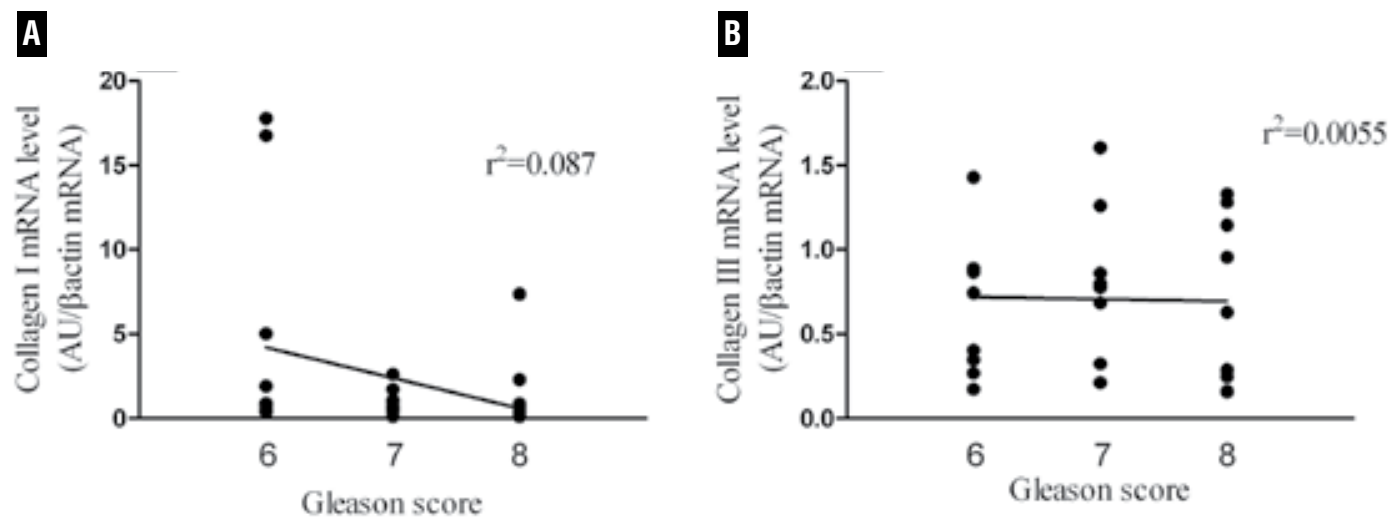

cancer, in all Gleason scores, when compared to benign areas, while metalloproteinase expression was significantly increased (Figure-4). The histological sections are shown in Figures 5, 6 and 7.

There was no correlation on the immunohistochemistry results of collagen I and III in relation to Gleason score in tumor areas. However, there was a positive correlation between metalloproteinase expression and Gleason score (Figure-8).

There was no statistical difference in gene and protein expression of both collagen I and III, as well as in metalloproteinase expression between tissues obtained from benign prostates from cystoprostatectomy and from benign prostatic tissues adjacent to the tumor area (Figure-9). Figure-10 shows that there was no difference or correlation in collagen expression related to pathological stage or preoperative PSA.

\section{DISCUSSION}

Since its description in 1966 by Donald Gleason (14), the Gleason grading has remained a cornerstone in the diagnosis and management of prostate cancer. However, the Gleason grading system is certainly not without limitations. A major limitation of the system is poor agreement between Gleason score in biopsies and prostatec- tomy specimens. In only one-third of the cases such agreement typically exists, with another one-third having a prostatectomy specimen score that is \pm 1 the score of a needle biopsy specimen. For the remaining one-third, the difference is 2 or more. Factors that contribute to such discrepancies include tumor heterogeneity, sampling errors, interchangeability and intrachangeability and interpretive errors. Surgical pathologists typically have a tendency to undergraduate the biopsy specimens (15-17). This fact raises the importance of development of new researches to evaluate other methods and factors that could improve the Gleason score diagnosis.

The concept that the microenvironment is crucial for the maintenance of cellular functions and tissue integrity suggests that a cancerinduced change in the stroma may contribute to cancer invasion (12).

It is known that MMPs such as type 1, 2 and 9 have been related to prostate cancer $(10,11)$. However, we have failed to find any previously published results related to collagen gene expression and prostate cancer. Based on the fact that collagen is one of the most abundant and important ECM components (18), the aim of this paper was to evaluate if the expression of metalloproteinase 13, collagen I and III could be related with pathological stage, PSA and Gleason score. 

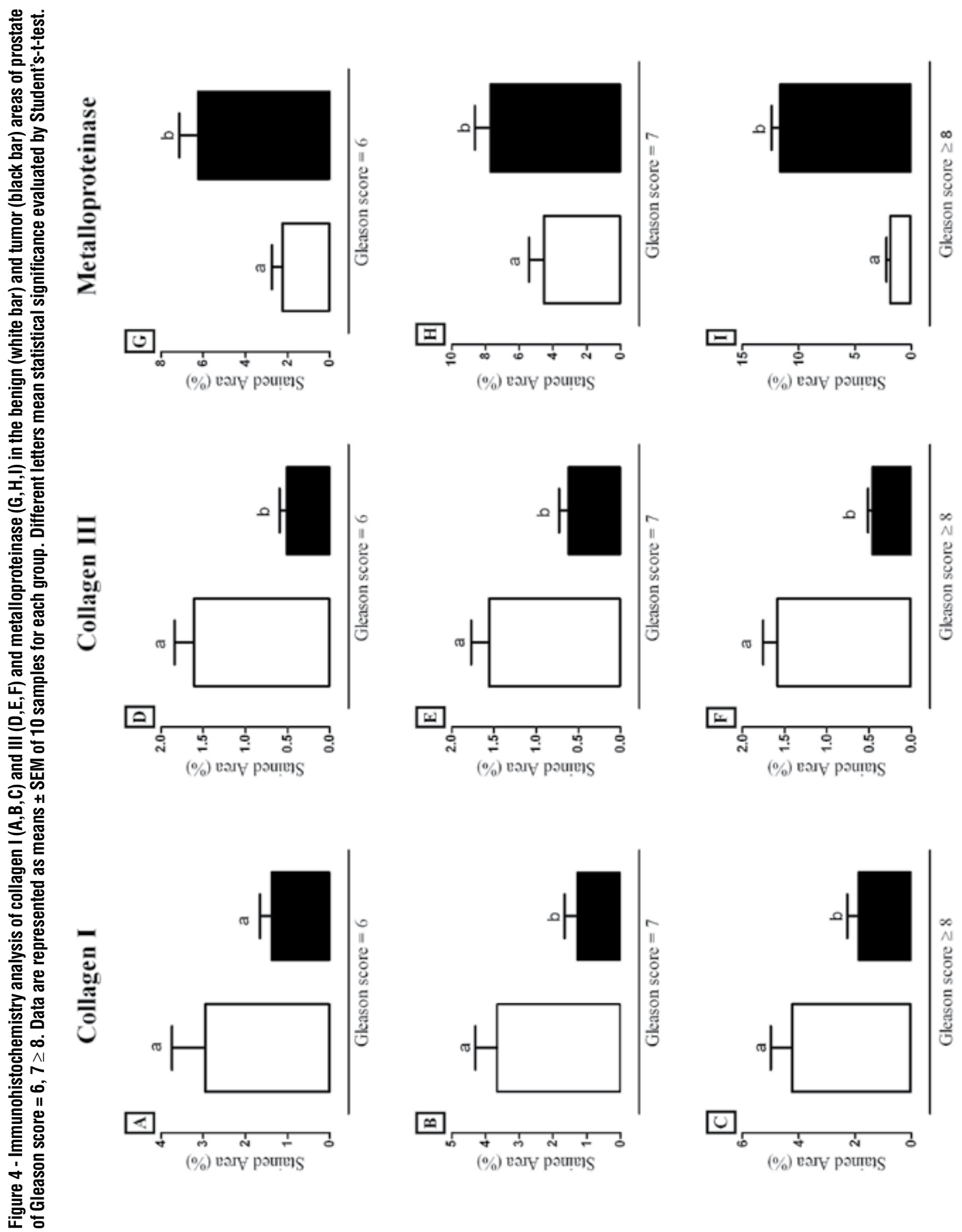
Figure 5 - Photomicrographs showing immunohistochemistry for benign and tumor areas for collagen I in prostate of Gleason score $\geq 8(A, E)$, Gleason score $=7(B, F)$ and Gleason score $=6(C, G)$. Prostate from patients with bladder cancer submitted to cystoprostatectomy (D). Negative controls (H). Final magnification 200x.

A

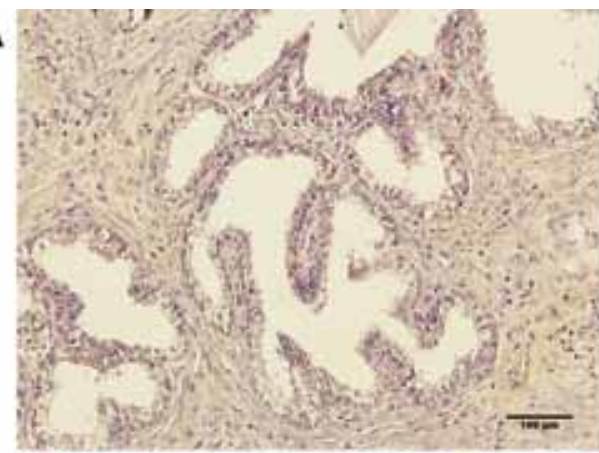

B

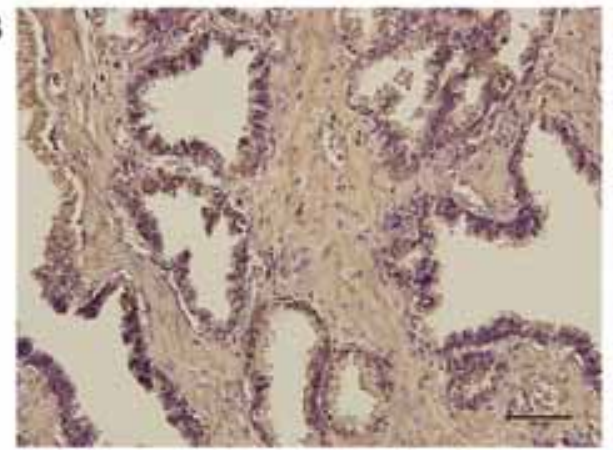

C

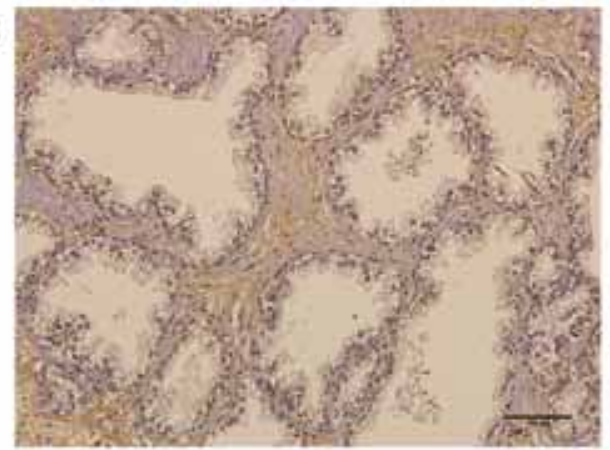

D

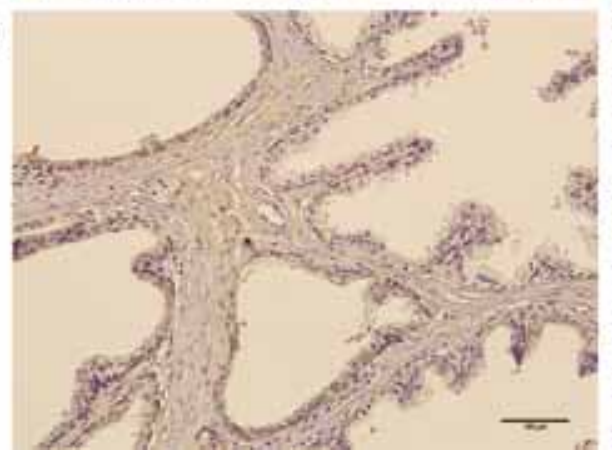

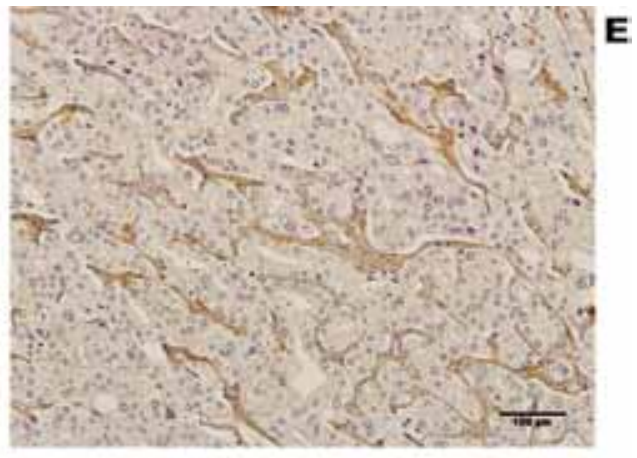
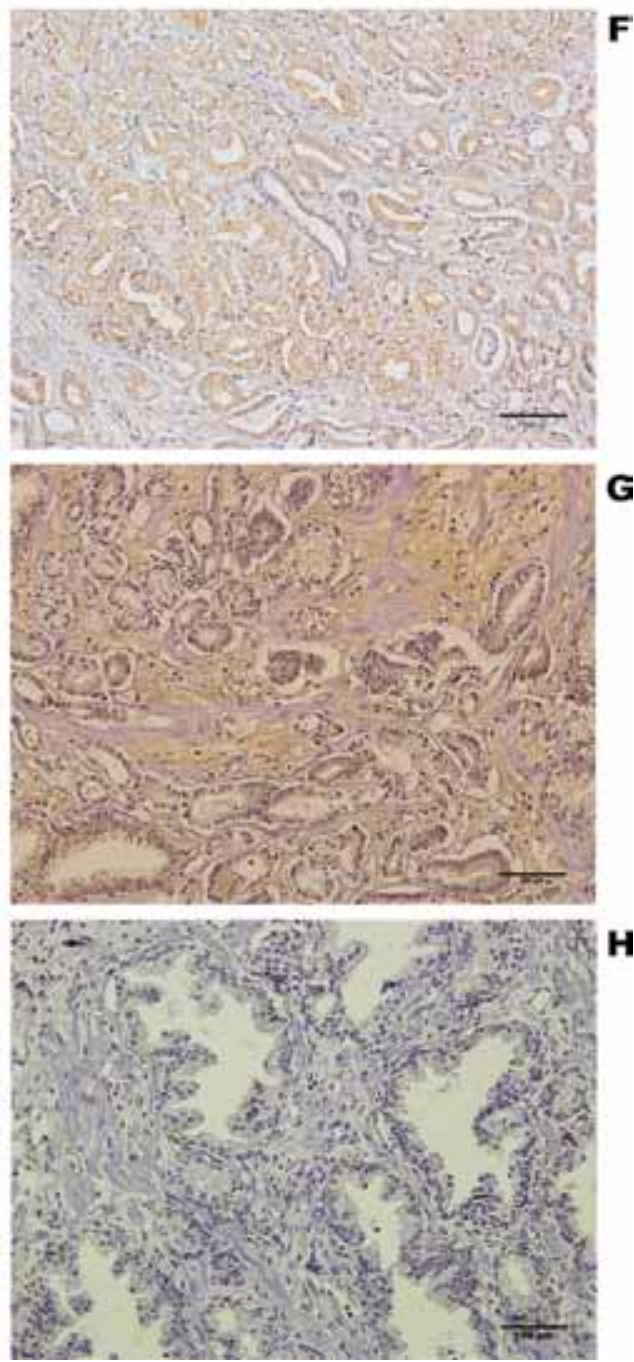
Figure 6 - Photomicrographs showing immunohistochemistry for benign and tumor areas for collagen III in prostate of Gleason score $\geq 8(A, E)$, Gleason score $=7(B, F)$ and Gleason score $=6(C, G)$. Prostate from patients with bladder cancer submitted to cystoprostatectomy (D). Negative controls (H). Final magnification 200x.

A

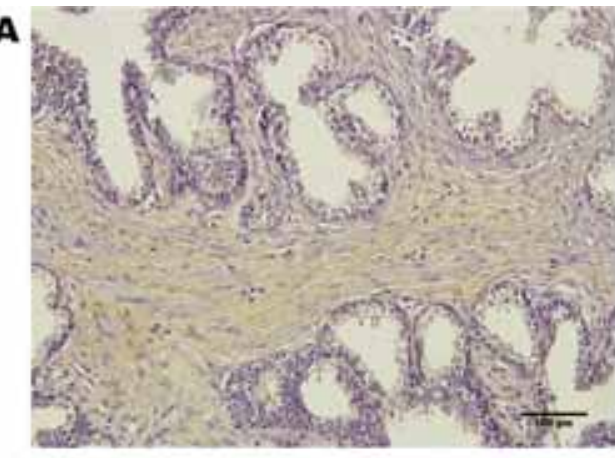

B

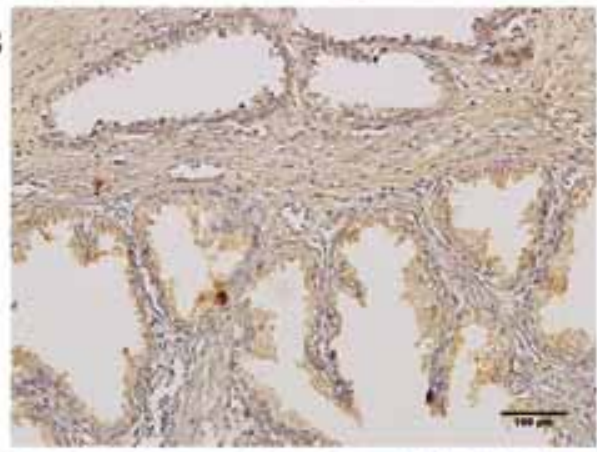

C

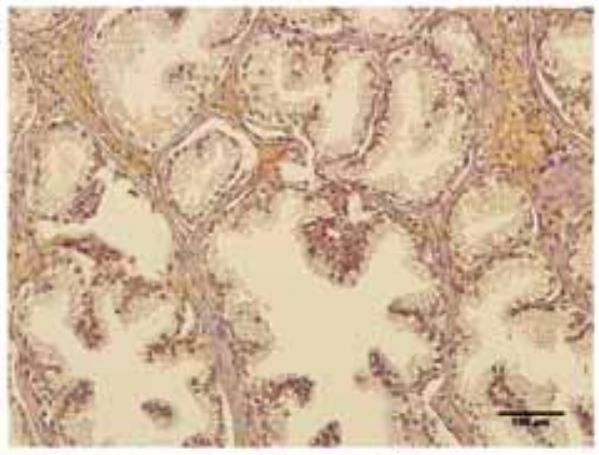

D

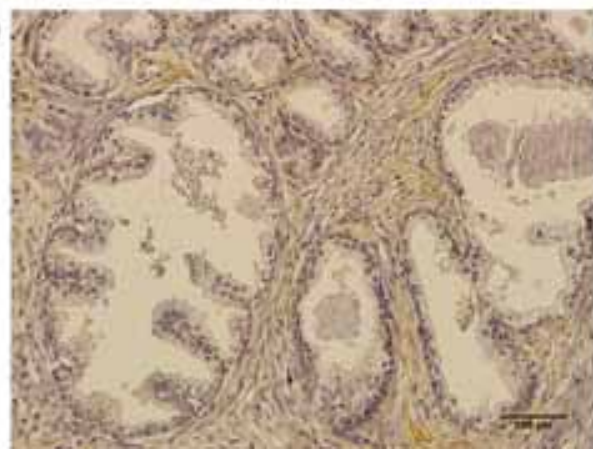

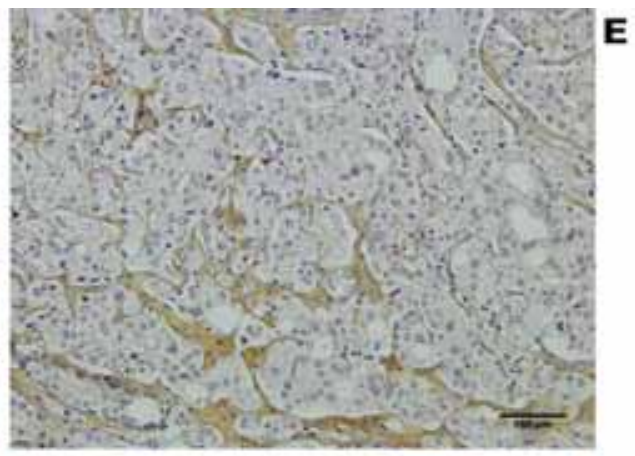

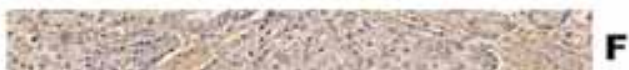

$\mathbf{F}$
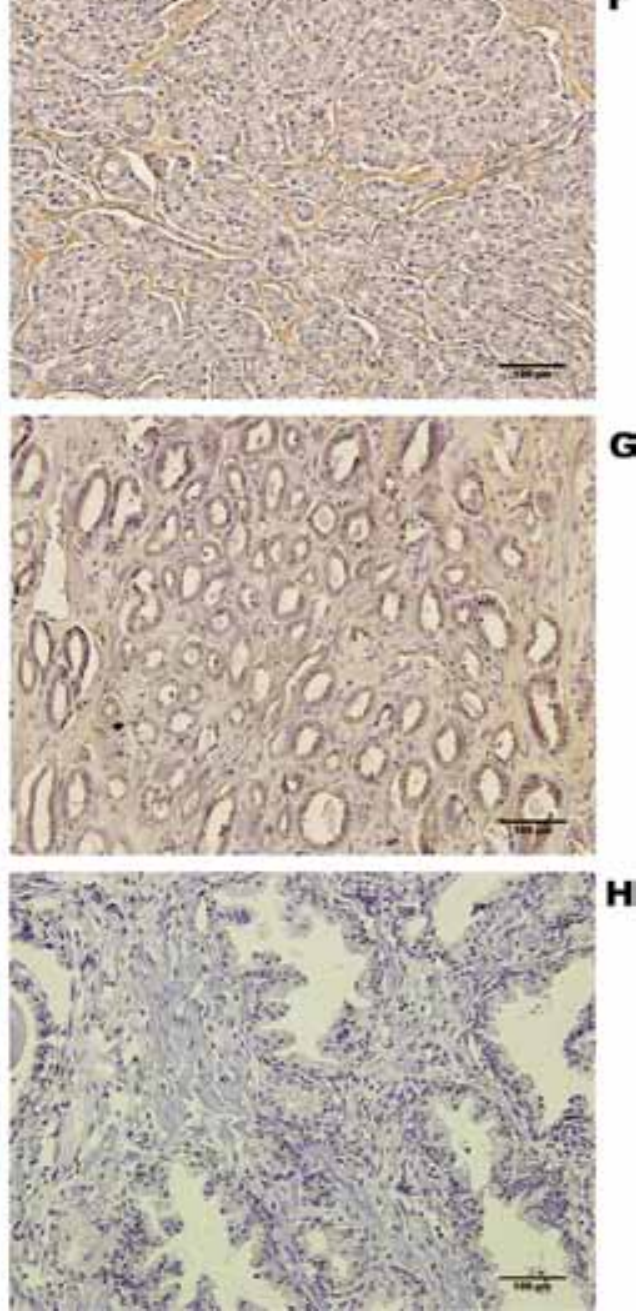
Figure 7 - Photomicrographs showing immunohistochemistry for benign and tumor areas for metalloproteinase in prostate of Gleason score $\geq 8(\mathrm{~A}, \mathrm{E})$, Gleason score $=7(\mathrm{~B}, \mathrm{~F})$ and Gleason score $=6(\mathrm{C}, \mathrm{G})$. Prostate from patients with bladder cancer submitted to cystoprostatectomy (D). Negative controls (H). Final magnification 200x.

A

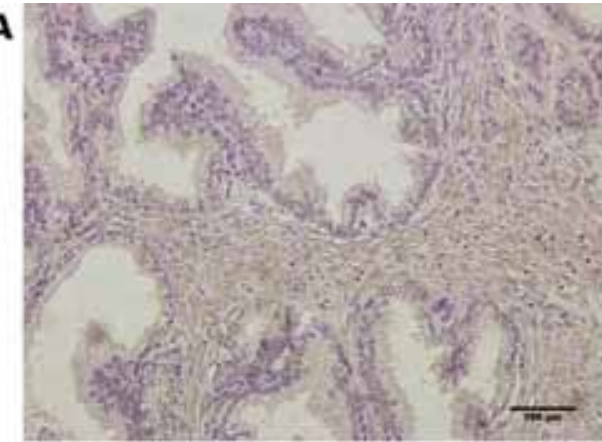

B

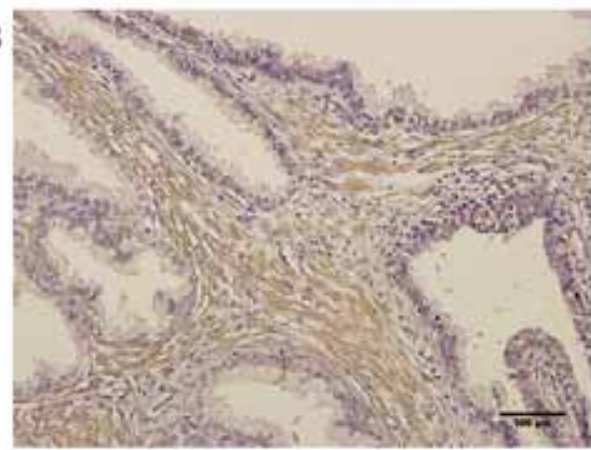

C

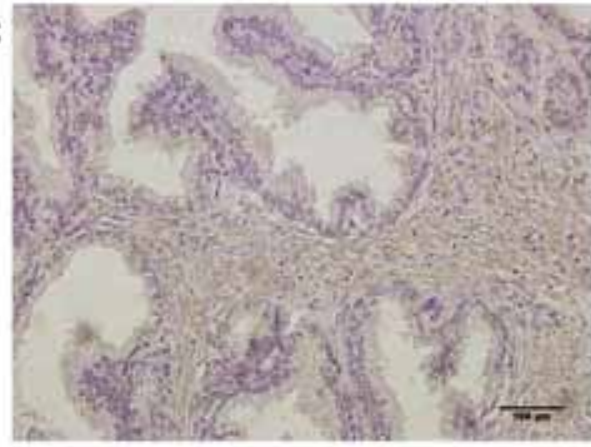

D

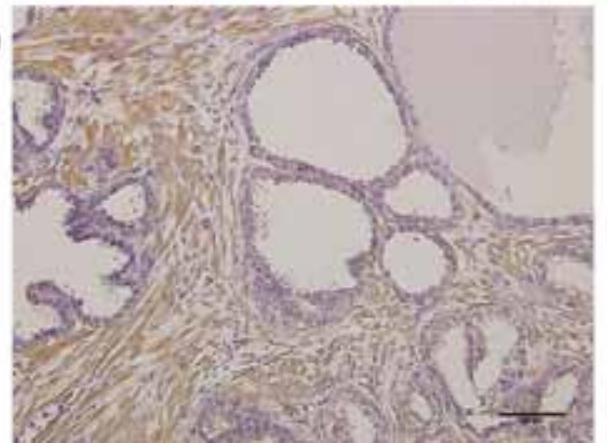

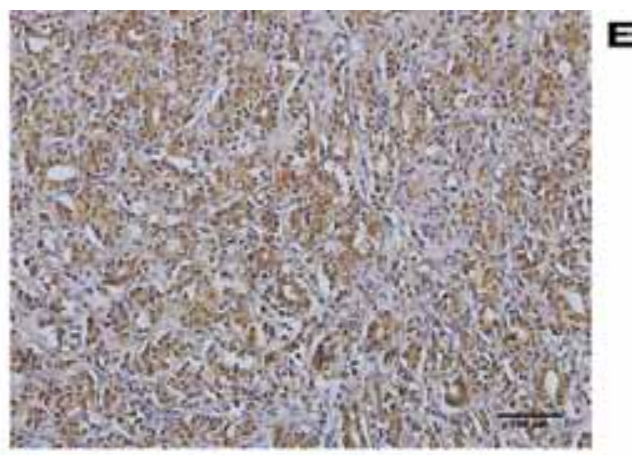
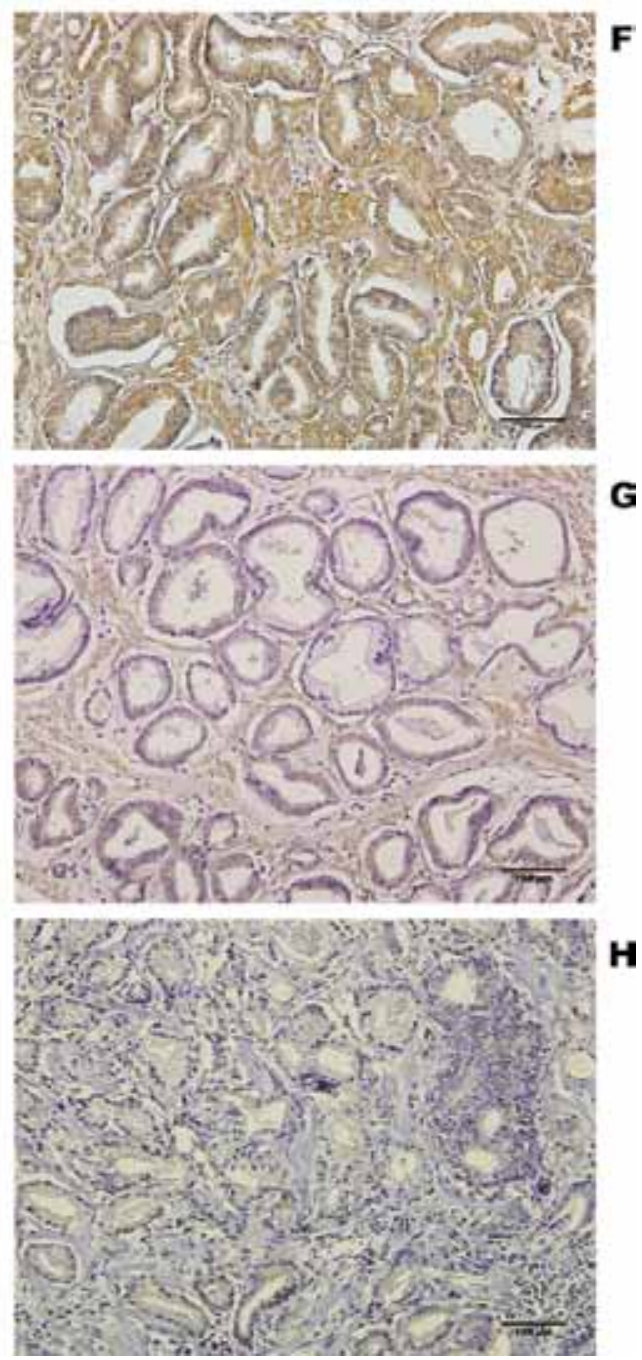
Figure 8 - Linear regression analysis between collagen I (A), III (B) and metalloproteinase (C) expression and Gleason score in tumor areas.
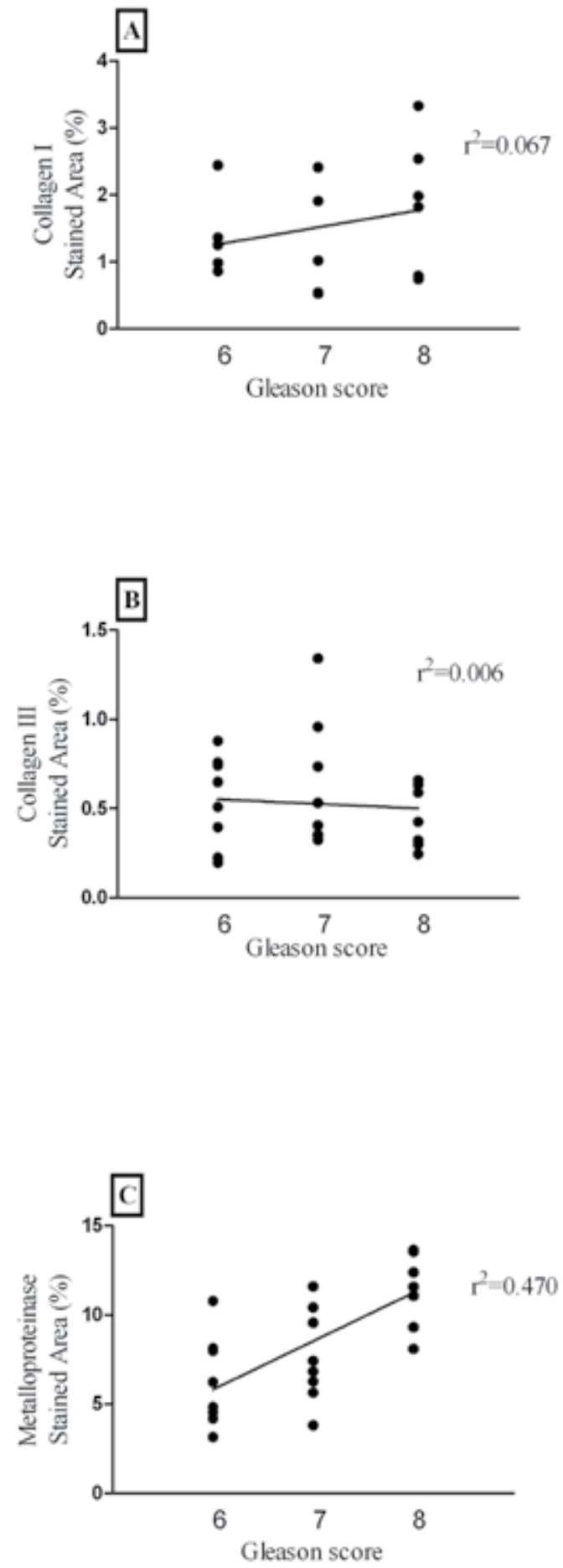
Figure 9 - Gene expression of Collagen I (A) and III (B) in the benign areas of cystoprostatectomy tissues and benign prostatic tissues adjacent to the tumor area in Gleason score $=6,7, \geq 8$. $\beta$ Actin was used as an internal control. Sample numbers were 13 for Gleason score=6; 10 for Gleason score=7 and 10 for Gleason score $\geq 8$. Protein expression of Collagen I (C), III (D) and Metalloproteinase $(E)$ in the benign areas of cystoprostatectomy tissues and benign prostatic tissues adjacent to the tumor area in Gleason score $=6,7, \geq 8$. Data are represented as means \pm SEM. Statistical significance was determined by ANOVA followed by Newman-Keuls test.
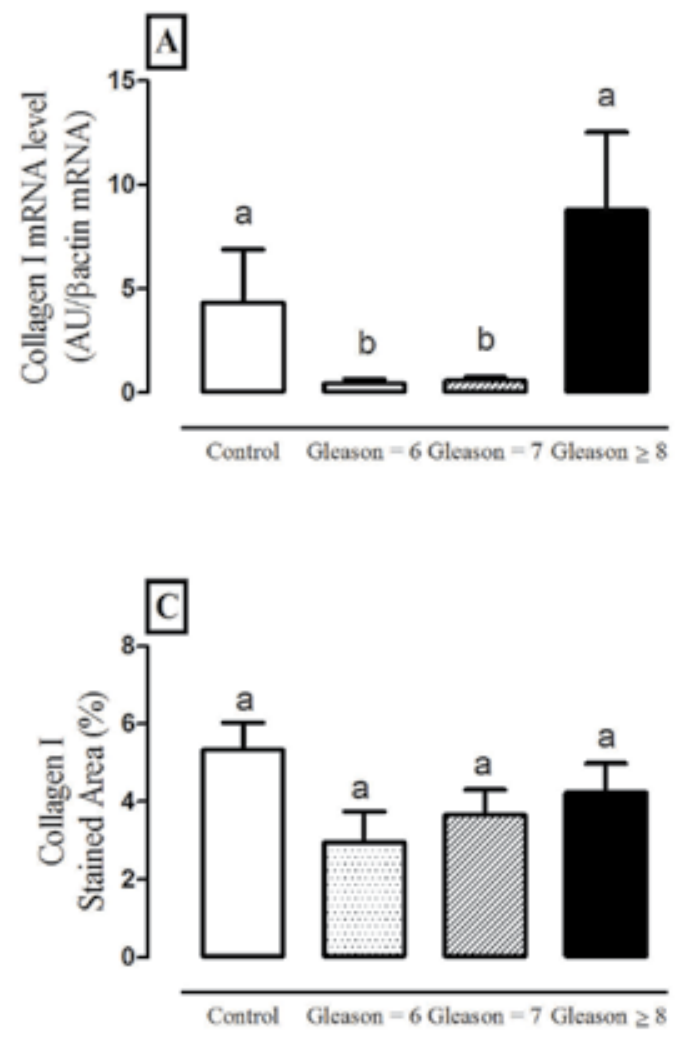
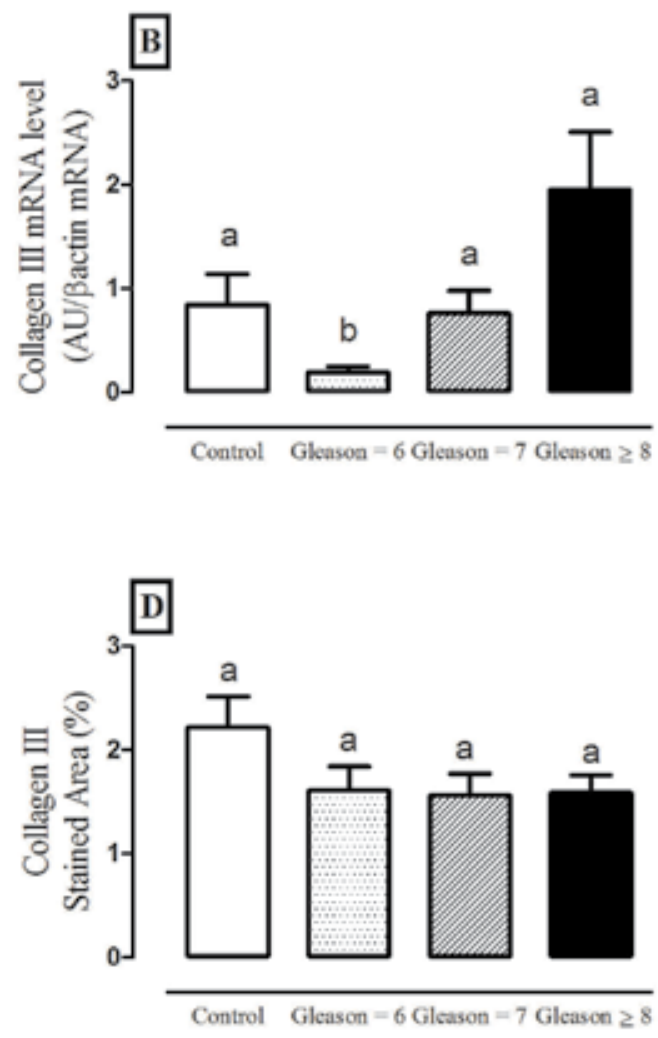

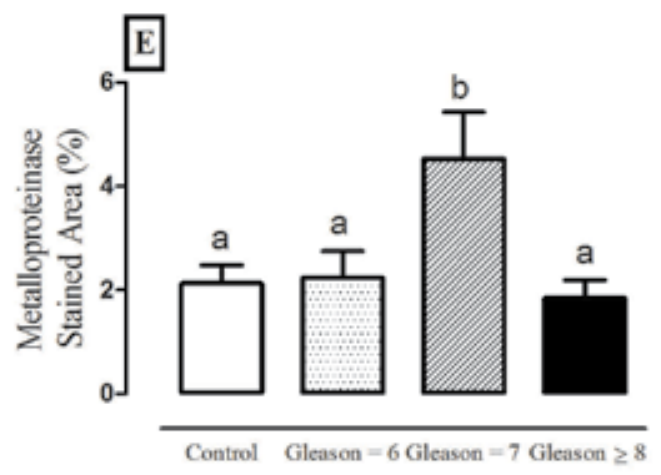


Figure 10 - Collagen I and III expression related to pathological stage (A,B) and Gleason score (C,D). Sample numbers were 13 for Gleason score=6; 10 for Gleason score=7 and 10 for Gleason score $\geq 8$. Data are represented as means \pm SEM.
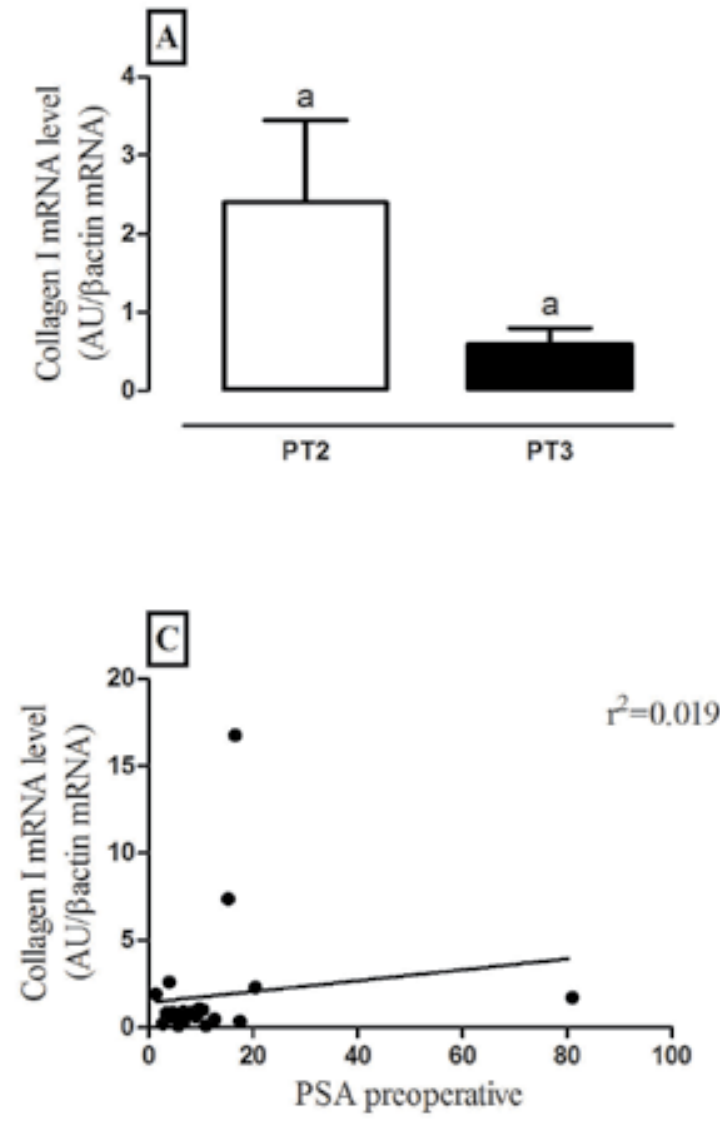
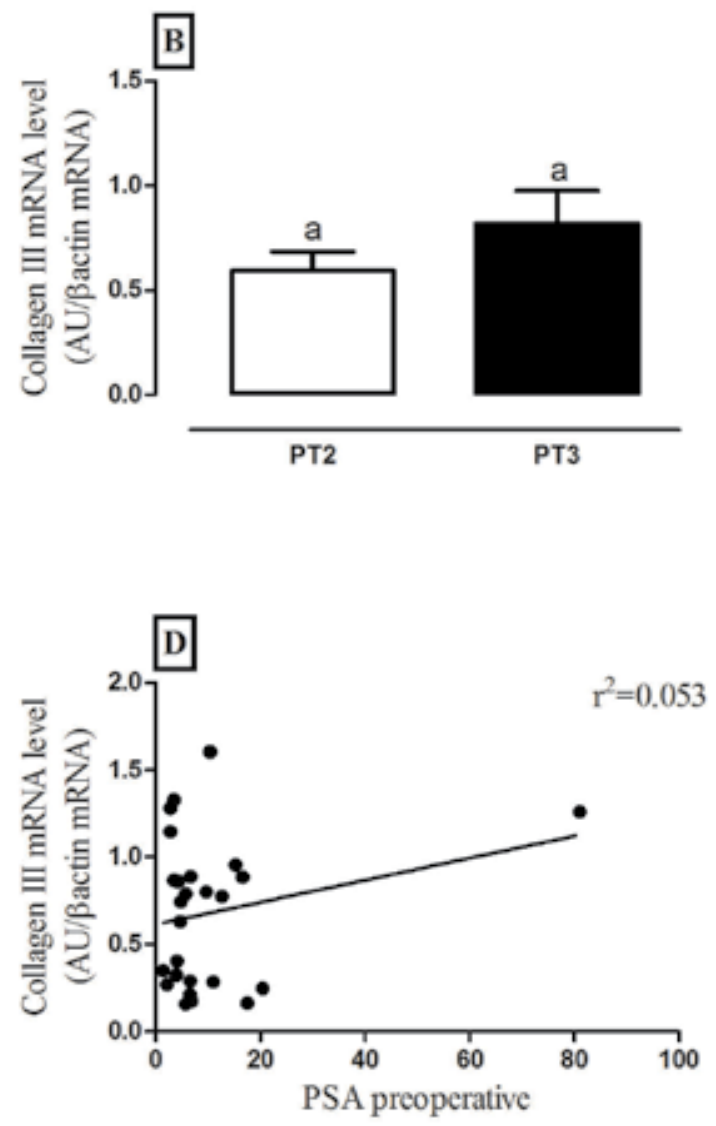

The results of long-term biochemical disease-free and cancer specific survival were evaluated in this study in relation to pathological stage, preoperative PSA and Gleason score. Despite the small number of patients, our sample is representative, since our results concerning patients' survival parameters are in agreement with another study from Johns Hopkins that evaluated 2409 patients from 1982 and 1999 (19).

The analysis of collagen I and III gene and protein expression showed different results. We can hypothesize that this discrepancy could be related to gene expression regulation mechanisms at RNA and protein levels. To try to clarify this hypothesis we evaluated the immune expres- sion of metalloproteinase protein that is responsible for ECM remodeling. The opposite results between metalloproteinase and collagen immune expression suggest that the collagen reduction in the tumor area could result from a high metalloproteinase activity. Despite the fact that we failed to find studies that have evaluated the metalloproteinase 13 , some papers have shown a high expression of metalloproteinase 1,2 and 9 in prostate cancer $(10,11)$.

Another possibility is that since the protein half time life is longer than the gene, we could hypothesize that the results observed in this study for the gene expression more recently occurred in the cells than those of proteins. 
Possibly a high metalloproteinase activity in tumor areas of all Gleason score was responsible for decreasing the collagen protein expression, in spite of a different gene regulation. Further experiments using cell culture are in progress to elucidate the gene regulation mechanisms in tumor areas.

Despite the lack of an explanation to the contradictory effects between gene and protein collagen expression, we failed to find other studies that have evaluated the collagen gene expression in prostate cancer with different Gleason scores, which, we believe, raises the importance of this study. We could also hypothesize that the difference in collagen expression between tumor and benign areas could have a functional relevance to the gland, the development and progression of the tumor.

Since there was no correlation between collagen expression and Gleason score at gene or protein level we can assume that collagen can not be used as a prognostic factor to improve Gleason score. However, the metalloproteinase immune expression showed a positive correlation with Gleason score. We strongly believe that the relation coefficient could be increased with the increment of samples. This data corroborate previous studies that evaluated different metalloproteinase proteins in prostate cancer $(10,11)$.

The lack of correlation between collagen and preoperative PSA could be related to the fact that high PSA is not prostate cancer specific. This fact also explains the lack of correlation between collagen and pathological stage since the correlation between Gleason score and pathological stage is well documented in the literature (20).

There is a great disagreement in the literature about the perfect control group to prostate samples. Some papers believe that the control group should be obtained from benign areas from the same patient while others strongly believe that the best control sample should come from a different patient since the adjacent areas could be somehow genetically modified. So, in this paper we used 2 control groups and the results showed that it does not exist a collagen distribution pattern among the different areas. So, we can assume that both areas could be used as a control group at least in relation to collagen expression.

We can conclude that while there is a positive correlation between metalloproteinase expression and Gleason score, this correlation is missed for collagen expression. So, while metalloproteinase may be a prognostic factor due to the association with the Gleason score, its expression and regulation do not seem to be related with collagen degradation.

\section{ACKNOWLEDGEMENTS}

This work was supported by the agencies CNPq (Brazilian Council of Science and Technology, www.cnpq.br) and FAPERJ (Rio de Janeiro State Foundation for Scientific Research, www. faperj.br).

The authors thank Carla B. M. Gallo for processing the images for this manuscript.

\section{CONFLICT OF INTEREST}

None declared.

\section{REFERENCES}

1. Condon MS: The role of the stromal microenvironment in prostate cancer. Semin Cancer Biol. 2005; 15: 132-7.

2. Noël A, Foidart JM: The role of stroma in breast carcinoma growth in vivo. J Mammary Gland Biol Neoplasia. 1998; 3: 215-25.

3. Martin M, Pujuguet P, Martin F: Role of stromal myofibroblasts infiltrating colon cancer in tumor invasion. Pathol Res Pract. 1996; 192: 712-7.

4. Tuxhorn JA, Ayala GE, Rowley DR: Reactive stroma in prostate cancer progression. J Urol. 2001; 166: 2472-83.

5. Tomas D, Kruslin B: The potential value of (Myo)fibroblastic stromal reaction in the diagnosis of prostatic adenocarcinoma. Prostate. 2004; 61: 324-31.

6. Rowley DR: What might a stromal response mean to prostate cancer progression? Cancer Metastasis Rev. 19981999; 17: 411-9.

7. Sappino AP, Schürch W, Gabbiani G: Differentiation repertoire of fibroblastic cells: expression of cytoskeletal proteins as marker of phenotypic modulations. Lab Invest. 1990; 63: 144-61. 
8. Hanamura N, Yoshida T, Matsumoto E, Kawarada Y, Sakakura T: Expression of fibronectin and tenascin- $C$ mRNA by myofibroblasts, vascular cells and epithelial cells in human colon adenomas and carcinomas. Int $\mathrm{J}$ Cancer. 1997; 73: 10-5.

9. Orimo A, Tomioka Y, Shimizu Y, Sato M, Oigawa S, Kamata $\mathrm{K}$, et al.: Cancer-associated myofibroblasts possess various factors to promote endometrial tumor progression. Clin Cancer Res. 2001; 7: 3097-105.

10. Trudel D, Fradet Y, Meyer F, Harel F, Têtu B: Membranetype-1 matrix metalloproteinase, matrix metalloproteinase 2 , and tissue inhibitor of matrix proteinase 2 in prostate cancer: identification of patients with poor prognosis by immunohistochemistry. Hum Pathol. 2008; 39: 731-9.

11. Trudel D, Fradet $Y$, Meyer F, Têtu B: Matrix metalloproteinase 9 is associated with Gleason score in prostate cancer but not with prognosis. Hum Pathol. 2010; 41: 1694-701.

12. Liotta LA, Kohn EC: The microenvironment of the tumourhost interface. Nature. 2001; 411: 375-9.

13. Lehr HA, van der Loos CM, Teeling P, Gown AM: Complete chromogen separation and analysis in double immunohistochemical stains using Photoshop-based image analysis. J Histochem Cytochem. 1999; 47: 119-26.

14. Gleason DF: Classification of prostatic carcinomas. Cancer Chemother Rep. 1966; 50: 125-8.

15. Bostwick DG: Gleason grading of prostatic needle biopsies. Correlation with grade in 316 matched prostatectomies. Am J Surg Pathol. 1994; 18: 796-803.
16. Cookson MS, Fleshner NE, Soloway SM, Fair WR: Correlation between Gleason score of needle biopsy and radical prostatectomy specimen: accuracy and clinical implications. J Urol. 1997 Feb; 157: 559-62.

17. Steinberg DM, Sauvageot J, Piantadosi S, Epstein JI: Correlation of prostate needle biopsy and radical prostatectomy Gleason grade in academic and community settings. Am J Surg Pathol. 1997; 21: 566-76.

18. Burns-Cox N, Avery NC, Gingell JC, Bailey AJ: Changes in collagen metabolism in prostate cancer: a host response that may alter progression. J Urol. 2001; 166: 1698-701.

19. Han M, Partin AW, Pound CR, Epstein JI, Walsh PC: Long-term biochemical disease-free and cancer-specific survival following anatomic radical retropubic prostatectomy. The 15-year Johns Hopkins experience. Urol Clin North Am. 2001; 28: 555-65.

20. Sim HG, Telesca D, Culp SH, Ellis WJ, Lange PH, True LD, et al.: Tertiary Gleason pattern 5 in Gleason 7 prostate cancer predicts pathological stage and biochemical recurrence. J Urol. 2008; 179: 1775-9.

\author{
Correspondence address: \\ Dr. Cristiane da Fonte Ramos \\ Urogenital Research Unit - UERJ \\ Avenida 28 de Setembro, 87 \\ Fundos - FCM - terreo, \\ Rio de Janeiro, RJ, 20561-030, Brazil \\ Fax: + $55212868-8033$ \\ E-mail: cramos_uerj@yahoo.com.br
}

\section{EDITORIAL COMMENT}

Several human cancers may induce a stromal reaction (desmoplasia) as a component of carcinoma progression. In cancers with stromal reaction, it seems that the response is similar, if not identical, to wound repair response. Prostate cancer may also be associated with wound repair type of reactive stroma composed of myofibroblasts and fibroblasts rather than normal prostate smooth muscle, which is displaced by the reactive stroma.
There is growing evidence that carcinogenesis is influenced and controlled by cellular interactions derived from a complex relationship between stromal, epithelial and extracellular matrix components. The neoplastic stromal environment is different from the stroma of the normal tissue and is characterized by modified extracellular matrix composition, increased microvessel density, inflammatory cells and myofibroblasts. Myofibroblasts in reactive stroma 
synthesize extracellular matrix components such as collagen I, collagen III, fibronectin, tenascin, and versican. In addition, myofibroblasts express proteases, fibroblast activation protein, and matrix metalloproteinases.

The authors found a positive correlation between metalloproteinase expression and Gleason score. The Gleason grading system is one of

\section{REFERENCE}

1. Epstein JI, Allsbrook WC Jr, Amin MB, Egevad LL; ISUP Grading Committee: The 2005 International Society of Urological Pathology (ISUP) Consensus Conference on Gleason Grading of Prostatic Carcinoma. Am J Surg Pathol. 2005; 29: 1228-42. the most important prognostic factors in prostate cancer and used all over the world. A revised version of the system was proposed by the International Society of Urological Pathology in 2005 (1). The results of the study favor that immunoexpression of metalloproteinase may be also a prognostic factor in prostate cancer due to the association with the Gleason score.
Dr. Athanase Billis

Full-Professor of Pathology State University of Campinas, Unicamp Campinas, São Paulo, Brazil E-mail: athanase@fcm.unicamp.br 